\title{
INEQUALITIES FOR POISSON KERNELS ON SYMMETRIC SPACES
}

\author{
ADAM KORÁNYI ${ }^{1}$
}

\begin{abstract}
Every symmetric space of noncompact type has a finite number of Furstenberg-Satake boundaries; to each of these there corresponds a Poisson kernel. Sharp Harnack-type inequalities are proved and it is shown that the Poisson kernel, in appropriate coordinates, is the square root of a rational function.
\end{abstract}

The purpose of this note is to prove sharp Harnack-type inequalities for various forms of the Poisson kernels of symmetric spaces of nonompact type. The case of the Cartan domains with their BergmanSilov boundary has already aroused some interest: The Harnack inequalities were proved for the classical domains by Tung [8], [9], and for domains of tube type, using a classification-free Jordan algebra method, by H. Resnikoff (unpublished). Here we will show (Proposition 2) that the case of any Cartan domain can easily be settled using standard Lie algebra machinery. Before that, in Proposition 1 we state more general sharp inequalities for the Poisson kernel of any symmetric space with respect to any of its Furstenberg-Satake boundaries. Finally, in Proposition 3, we obtain more precise information about the Poisson kernel expressed in orispherical coordinates; this result also leads to a simplified proof of an estimate used by L. Lindahl [6]. Our basic techniques throughout are those of Harish-Chandra [1]; we also make use of a relatively simple lemma of Lindahl [6].

1. We follow the notations of [5]. $X=G / K$ is a Riemannian symmetric space of noncompact type, $\mathfrak{g}=\mathfrak{f}+\mathfrak{p}$ the corresponding Cartan decomposition of the Lie algebra, a maximal Abelian in $\mathfrak{p}, \mathfrak{t} \supset \mathfrak{a}$ a Cartan subalgebra of $g$. The restricted roots (restrictions of the $t^{c}$-roots of $g^{c}$ to $a$ ) form a root system, $F$ is a corresponding simple system of roots, $a^{+}$the positive Weyl chamber. Given a subset $E \subset F, \mathfrak{a}(E)$ is the subspace of $\mathfrak{a}$ annihilated by all $\lambda \in E, 2 \rho_{E}$ is the sum of those positive restricted roots

Received by the editors June 12, 1973.

AMS (MOS) subject classifications (1970). Primary 22E30; Secondary 43A85.

Key words and phrases. Symmetric spaces, Furstenberg-Satake boundaries, bounded symmetric domains.

${ }^{1}$ Partially supported by NSF grant GP-28448.

(c) American Mathematical Society 1974 
which do not vanish on $a(E)$. To $E$ there corresponds a parabolic subgroup $B(E)$ of $G$ and a Furstenberg-Satake boundary $G / B(E)$, on which $K$ acts transitively. The corresponding Poisson kernel is given [4, Proposition 1.2] for $g \in G, k \in K, k \in G / B(E)$, by

$$
P_{E}(g \cdot o, k)=e^{-2 \rho_{E}\left(H\left(g^{-1} k\right)\right)}
$$

where 0 is the identity coset in $G / K$ and $H(g)$ is defined by $g=k(g)$. $\exp H(g) \cdot n(g)$ (Iwasawa decomposition).

Every point in $X$ can be written ("in polar coordinates") as $k_{1} a \cdot o$ with $k_{1} \in K, a \in A^{+}=\exp a^{+}$. Let $W$ be the small Weyl group and let $s_{0}$ be the element in it that maps $\mathfrak{a}^{+}$onto $-\mathfrak{a}^{+}$. The following is a general form of the Harnack inequalities:

Proposition 1. Let $a \in A^{+}$be fixed. For $k_{1}, k_{2} \in K$ we have the sharp inequalities

$$
\exp \left\{2 \rho_{E}\left(s_{0}(\log a)\right)\right\} \leqq P_{E}\left(k_{1} a \cdot o, k_{2}\right) \leqq \exp \left\{2 \rho_{E}(\log a)\right\} .
$$

When $E=\varnothing$ or when $s_{0}=-\mathrm{id}$, not however in the general case, the lower bound is also equal to $\exp \left\{-2 \rho_{E}(\log a)\right\}$.

Proof. The inequalities can be deduced from [1, Lemma 35] and [6, Lemma 5.1]; we give here a simplified proof extracting the relevant part from Harish-Chandra's argument [1]. By [6, Lemma 5.1], $2 \rho_{E}$ is the restriction to $\mathfrak{a}$ of the highest weight of a finite dimensional irreducible representation $\pi$ of $G^{c}$, the simply connected complexification of $G$. Let $\varphi$ be a highest weight vector of unit length (we use an inner product for which $\pi(A)$ is Hermitian, $\pi(K)$ is unitary). Now $\pi(N) \varphi=\varphi$, and the smallest eigenvalue of the positive operator $\pi\left(a^{-1}\right)$ is equal to the reciprocal of the largest eigenvalue of $\pi(a)$, i.e. to $\exp \left\{-2 \rho_{E}(\log a)\right\}$. Writing $k=k_{1}^{-1} k_{2}$ it follows that

$$
\exp \left\{2 \rho_{E}\left(H\left(a^{-1} k\right)\right)\right\}=\left\|\pi\left(a^{-1} k\right) \varphi\right\| \geqq \exp \left\{-2 \rho_{E}(\log a)\right\}
$$

which gives the desired upper bound. It is sharp, since equality holds for $k=e$.

To get the lower bound let $k_{0} \in K$ be such that $s_{0}=\left.\operatorname{ad}\left(k_{0}\right)\right|_{\mathrm{a}}$. Then we have

$$
\begin{aligned}
\exp \left\{2 \rho_{E}\left(H\left(a^{-1} k\right)\right)\right\} & =\exp \left\{2 \rho_{E}\left(H\left(s_{0}\left(a^{-1}\right) k_{0} k\right)\right)\right\} \\
& =\left\|\pi\left(s_{0}\left(a^{-1}\right)\right) \pi\left(k_{0} k\right) \varphi\right\| \leqq \exp \left\{-2 \rho_{E}\left(s_{0}(\log a)\right)\right\}
\end{aligned}
$$

since the last expression gives the largest eigenvalue of $\pi\left(s_{0}\left(a^{-1}\right)\right)$. Equality holds for $k=k_{0}^{-1}$, i.e. for $k_{1}=k_{2} k_{0}$. 
The statement about the other expression for the lower bound follows in the case $E=\varnothing$ by remarking that $-s_{0}$ is a permutation of $F$, and so $\rho\left(s_{0} H\right)=-\rho(H)$ for all $H \in \mathfrak{a}$ (cf. [1]). In the case $s_{0}=-$ id it is trivially true for any $E$. That it is not always true can be seen by considering the special case $X=S L(3, R) / S O(3)$ (restricted root system $A_{2}$ ) with $E$ consisting of a single element of $F$. This finishes the proof.

We want to specialize our result to the case of a Hermitian symmetric space given as a bounded domain $\mathscr{D}$ in the canonical Harish-Chandra realization. By an obvious multiplicative property it is enough to consider the case where $\mathscr{D}$ is irreducible.

As is well known (e.g. [2, Chapter VIII, §7]) now g has a Cartan subalgebra $\mathfrak{h}$ contained in $\mathfrak{f}$. For any $\mathfrak{h}^{c}$-root $\alpha$, let $E_{\alpha}, H_{\alpha}$ denote the usual basis elements of $\mathfrak{g}^{c} \cdot \mathfrak{g}^{c}$ has a certain subspace $\mathfrak{p}^{-}$which is a complex Euclidean space with $\operatorname{Ad}(K)$ acting on it unitarily; $\mathscr{D}$ is realized as a domain in $\mathfrak{p}^{-}$. There is a set $\Psi$ of "strongly orthogonal" positive roots such that $\mathscr{D}$ is equal to the set of points $\operatorname{Ad}(k) \sum_{\psi \in \Psi} r_{\psi} E_{-\psi}\left(0 \leqq r_{\psi}<1\right.$, $k \in K)$. The Bergman-Šilov boundary $\mathscr{B}$ of $\mathscr{D}$ is the orbit of $\sum_{\psi} E_{-\psi}$ under $\operatorname{Ad}(K)$. The Poisson kernel [3], written $\mathscr{P}(z, u)$, is a function on $\mathscr{D} \times \mathscr{B}$. The Harnack inequalities now take the following form.

Proposition 2. Let $\mathscr{D}$ be irreducible, of (complex) dimension $n$ and of rank l. Let $z=\operatorname{Ad}(k) \sum r_{\psi} E_{\psi}\left(0 \leqq r_{\psi}<1\right)$. Then

$$
\prod_{\psi \in \Psi}\left(\frac{1-r_{\psi}}{1+r_{\psi}}\right)^{n / l} \leqq \mathscr{P}(z, u) \leqq \prod_{\psi \in \Psi}\left(\frac{1+r_{\psi}}{1-r_{\psi}}\right)^{n / l}
$$

for all $k \in K, u \in \mathscr{B}$. These inequalities are sharp.

Proof. By Harish-Chandra's results (cf. [2, Chapter VIII]) $a=$ $\sum \boldsymbol{R}\left(E_{\psi}+E_{-\psi}\right)$ is a maximal Abelian subspace in $\mathfrak{p}$, and $z$ corresponds to the element $k a \cdot o$ of $G / K$ with $a=\exp \sum t_{\psi}\left(E_{\psi}+E_{-\psi}\right), r_{\psi}=\tanh t_{\psi}$. As proved by Moore [7] (and also briefly described in [5, p. 403]) there is an automorphism of $\mathrm{g}^{c}$ (the Cayley transform) which carries each $E_{\psi}+E_{-\psi}$ to $H_{\psi}$; it carries the restricted roots to the restrictions of the $\mathfrak{h}^{c}$-roots to $\mathfrak{h}^{-}=\sum \boldsymbol{R} H_{\psi}$. Writing $\Psi=\left\{\psi_{1}, \cdots, \psi_{l}\right\}$, these restrictions are of the form $\pm \psi_{j}$ (each with multiplicity one), $\pm \frac{1}{2}\left(\psi_{j} \pm \psi_{k}\right)(j<k$, each with the same multiplicity $a>0$ ), $\pm \frac{1}{2} \psi_{j}$ (with multiplicity $2 b \geqq 0$ ). $F$ can be chosen as the set of all $\frac{1}{2}\left(\psi_{j}-\psi_{j+1}\right)(1 \leqq j \leqq l-1)$ together with $\psi_{l}$ or $\frac{1}{2} \psi_{l}$ depending on whether $b$ is zero or not; the subset $E$ consisting of all the $\frac{1}{2}\left(\psi_{j}-\psi_{j+1}\right)$ gives a $G / B(E)$ isomorphic with $\mathscr{B}$.

There are now two ways of proving our assertions. One is to use [3, Proposition 5.7] to compute $\mathscr{P}(z, u)$ for $z \sim a \cdot o, u \sim \dot{e}$ and $k_{0}$; from the proof of Proposition 1 we know that these values are the desired sharp bounds. The more interesting way, independent of [3] and still easy, 
is to explicitly evaluate $2 \rho_{E}(\log a)$. For this we have to note that $a(E)$ is one-dimensional, and the Cayley transform carries it to the line spanned by $\sum_{\psi \in \Psi} H_{\psi}$. The positive restricted roots that do not vanish on this line are $\psi_{j}(1 \leqq j \leqq l), \frac{1}{2}\left(\psi_{j}+\psi_{k}\right)(j>k)$ and $\frac{1}{2} \psi_{j}$; their sum (with multiplicities) is equal to $(1+((l-1) / 2) a+b) \sum_{\psi \in \Psi} \psi$. Evaluating this on $\sum t_{\psi} H_{\psi}$ gives $2 \rho_{E}(\log a)=(2+(l+1) a+2 b) \sum t_{\psi}$. Making use of the fact [7] that $\mathfrak{p}^{-}$is spanned by certain of $E_{-\alpha}$ and the restrictions of the $\alpha$ that occur are $\psi_{j}, \frac{1}{2}\left(\psi_{j}+\psi_{k}\right)\left(j<k, a\right.$ times) $\frac{1}{2} \psi_{j}$ ( $b$ times) we see that this is also equal to $2 n / l \sum t_{\psi}$. Now $r=\tanh t$ implies $e^{2 t}=(1+r)(1-r)^{-1}$ and our formula for the upper bound follows. The lower bound is its reciprocal since $-\mathrm{id} \in W$, as one sees from the explicit description of the restricted root system.

Remark. The Szegö kernel $\mathscr{S}(z, u)$ of $\mathscr{D}$ and the Poisson kernel are in the following simple relationship:

$$
\mathscr{P}(z, u)=|\mathscr{S}(z, u)|^{2} / \mathscr{S}(z, z)
$$

and it is known [3, Proposition 5.7] that $\mathscr{S}(z, z)=\Pi\left(1-r_{\psi}^{2}\right)^{-n / l}$. It follows immediately that, for $z, u$ as in Proposition 2, we have the sharp inequalities

$$
\Pi\left(1+r_{\psi}^{2}\right)^{-n / l} \leqq|\mathscr{S}(z, u)| \leqq \Pi\left(1-r_{\psi}^{2}\right)^{-n / l} .
$$

Analogous inequalities hold for the Bergman kernel, which is proportional to a positive power of the Szegö kernel.

2. Returning to the case of an arbitrary symmetric space, let $\overline{\mathrm{n}}(E)^{c}$ be the sum of all $t^{c}$-root spaces $g_{-\alpha}^{c}$ such that $\alpha>0, \alpha \mid a(E) \neq 0$. Let $\overline{\mathrm{n}}(E)=\overline{\mathrm{n}}(E)^{c} \cap \mathrm{g}$. The function $\psi_{E}(\bar{n})=\exp \left\{-2 \rho_{E}(H(\bar{n}))\right\}$ defined for $\bar{n} \in \bar{N}(E)=\exp \bar{n}(E)$ is just another form of the Poisson kernel and plays an important role in proving Fatou-type theorems [5], [6]. We shall prove that $\psi_{\bar{E}}^{-2}$, and even $\psi_{\bar{E}}^{-1}$ if $E=\varnothing$, is a sum of squares of polynomials (in canonical coordinates) and assumes its minimum value 1 only when $\bar{n}=e$. On one hand, this can be regarded as a sharp Harnack inequality. On the other, looking at behavior at infinity, this implies the key estimates of Lindahl [6, Lemmas 5.2, 5.3] by an obvious compactness argument.

Just as in Lindahl's case all that is needed is that $2 \rho_{E}$, resp. $\rho_{\varnothing}$, is the restriction of a highest weight with certain properties.

Proposition 3. Let $\omega$ be the highest weight of a finite-dimensional irreducible representation $\pi$ such that $\omega\left(H_{\alpha}\right)>0$ for all simple $\mathrm{t}^{c}$-roots $\alpha$ that do not vanish on $\mathfrak{a}(E)$. Then, for all $\bar{n} \in \bar{N}(E)$,

$$
\exp \{2 \omega(H(\bar{n}))\}=1+\sum_{j}\left|P_{j}(\bar{n})\right|^{2}
$$

with a finite number of polynomials $P_{j}$ whose only common zero is at $\bar{n}=e$. 
Proof. We use an inner product on the representation space as in Proposition 1 and take an orthonormal basis $\left\{\varphi_{1}, \cdots, \varphi_{n}\right\}$ such that each $\varphi_{j}$ is in the weight space for some weight $\omega_{j}$ and $\omega_{j} \geqq \omega_{k}$ for $j>k$. So $\varphi=\varphi_{1}$ is a highest weight vector. $\overline{\mathrm{n}}(E)$ is represented by strict triangular matrices, $\bar{N}(E)$ by unipotent matrices. Each matrix entry $\pi(\bar{n})_{j k}$ is a polynomial in $\bar{n} \in \bar{N}(E)$, since the Campbell-Hausdorff formula is finite on nilpotent Lie algebras. We have

$$
e^{2 \omega(H(\bar{n}))}=\|\pi(\bar{n}) \varphi\|^{2}=\left\|\sum_{j} \pi(\bar{n})_{j 1} \varphi_{j}\right\|^{2}=\sum_{j}\left|\pi(\bar{n})_{j 1}\right|^{2} .
$$

Now $\pi(\bar{n})_{11}=1$ since $\pi(\bar{n})$ is unipotent; $P_{j}(\bar{n})=\pi(\bar{n})_{j 1}(j>1)$ are polynomials with $P_{j}(e)=0$, since $\pi(e)=$ id. We have to show only that $P_{j}(\bar{n})=0$ for all $j>1$ implies $\bar{n}=e$, i.e. that $\pi(\bar{n}) \varphi=\varphi$ implies $\bar{n}=e$.

Assume $\pi(\bar{n}) \varphi=\varphi$. Writing $\bar{n}=\exp X \quad(X \in \bar{n}(E))$ we have that $\exp t \pi(X) \varphi-\varphi$ is a (vector-valued) polynomial in $t$ vanishing for $t=0,1$, $2, \cdots$, hence identically zero. It follows that $\pi(X) \varphi=0$; we must show that $X=0$. Taking a basis $\left\{X_{-\alpha}\right\}$ of $\overline{\mathrm{n}}(E)$ such that $X_{-\alpha} \in \mathrm{g}_{-\alpha}^{c}\left(\alpha>0,\left.\alpha\right|_{a(E)} \neq\right.$ 0 ) it suffices to show that $\pi\left(X_{-\alpha}\right) \varphi \neq 0$ for each $\alpha$. Now it is well known that the vectors $\pi\left(X_{-\alpha}\right)^{j} \varphi$ for $0 \leqq j \leqq \omega\left(H_{\alpha}\right)$ form a basis of an irreducible module for the three-dimensional Lie algebra generated by $\left\{X_{-\alpha}, H_{\alpha}, X_{\alpha}\right\}$. So $\pi\left(X_{-\alpha}\right) \varphi \neq 0$ when $\omega\left(H_{\alpha}\right)>0$; this however follows from our hypothesis by writing $\alpha$ as a combination of simple roots.

\section{REFERENCES}

1. Harish-Chandra, Spherical functions on a semisimple Lie group. I, Amer. J. Math. 80 (1958), 241-310. MR 20 \#925.

2. S. Helgason, Differential geometry and symmetric spaces, Pure and Appl. Math., vol. 12, Academic Press, New York, 1962. MR 26 \#2986.

3. A. Korányi, The Poisson integral for generalized half-planes and bounded symmetric domains, Ann. of Math. (2) 82 (1965), 332-350. MR 34 \#371.

4. - Boundary behavior of Poisson integrals on symmetric spaces, Trans. Amer. Math. Soc. 140 (1969), 393-409. MR 39 \#7132.

5. - Harmonic functions on symmetric spaces, in Symmetric spaces, Boothby and Weiss (editors), Dekker, New York, 1972.

6. L. Lindahl, Fatou's theorem for symmetric spaces, Ark. Mat. 10 (1972), 33-47.

7. C. C. Moore, Compactifications of symmetric spaces. II: The Cartan domains, Amer. J. Math. 86 (1964), 358-378. MR 28 \#5147.

8. S. H. Tung, Harnack's inequality and theorems on matrix spaces, Proc. Amer. Math. Soc. 15 (1964), 375-381. MR 28 \#4135.

9. - Harnack's in equalities on the classical Cartan domains, Pacific J. Math. 16 (1966), 373-378. MR 32 \#7789.

Belfer Graduate School of Science, Yeshiva University, New York, New YORK 10033 